\title{
Prevalence and incidence of venous leg ulcers - a protocol for a systematic review
}

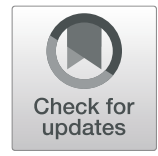

S. Probst ${ }^{*}$ (D, C. D. Weller ${ }^{2}$, P. Bobbink ${ }^{3}$, C. Saini ${ }^{3}$, M. Pugliese ${ }^{3}$, Monika Buehrer Skinner $^{4}$ and G. Gethin ${ }^{5}$

\begin{abstract}
Background: Venous leg ulcers (VLUs) are chronic wounds characterized by slow healing and high recurrence. Information on prevalence and incidence is essential for ascertaining the burden of VLU on the health care system and to inform epidemiological research, priority setting, and health care planning. The objective of this protocol is to present a transparent process for how we plan to review the existing international literature on the prevalence and incidence of VLU as well as the characteristics of the population reported within these studies.

Methods: An exploratory search was performed using MEDLINE via PubMed and CINHAL via Ebsco to identify concepts, keywords, MeSH terms, and headings to identify study types looking at data of VLU prevalence and/or incidence and related patient characteristics. The findings of this exploratory search will determine the final search strategy. The titles and abstracts of the identified articles will be screened independently be two authors for relevance. Study which pass the quality assessment will be included. Data extraction will be performed independently by two authors and in accordance with a pre-designed data extraction form. If the data allows, a meta-analysis will be performed otherwise a descriptive summary of the findings will be conducted.

Discussion: The results of this review will contribute to the evidence base on VLU occurrence and may inform the decision making of healthcare professionals, policy-makers, and consumers. It will also inform future research in this area of VLU care.
\end{abstract}

Systematic review registration: PROSPERO CRD42020205855

Keywords: Venous leg ulcer, Prevalence, Incidence, Epidemiology

\section{Background}

Venous leg ulcers (VLUs) are open lesions of the lower limb and represent between 60 and $80 \%$ of all leg ulcerations that occur in the presence of venous disease $[1,2]$. Healing rates are protracted with only $60 \%$ on average healed by 12 weeks, and once healed, 75\% develop a recurrence within 3 weeks [3]. At least $60 \%$ of VLUs result in a chronic wound [4]. VLUs are most prevalent among persons of older age with concomitant chronic venous insufficiency. They impact more females than males, those who are obese, immobile, have a congenital

\footnotetext{
* Correspondence: sebastian.probst@hesge.ch

${ }^{1}$ HES-SO University of Applied Sciences and Arts Western Switzerland, School of Health Sciences, Avenue de Champel 47, 1206 Geneva, Switzerland

Full list of author information is available at the end of the article
}

absence of veins, or a history of deep vein thrombosis (DVT) or phlebitis [5] resulting in reduced mobility, poor quality of life, and notable financial burden on patients and health care systems [6]. Data from Australia estimate the annual health care costs to VLU treatment of more than AUD\$ 3 billion yearly [7] and in the UK at £941 million [8].

Three VLU studies have reported prevalence and incidence of populations in various settings ranging for prevalence from $0.12 \%$ [9] to $1.69 \%$ [10] and for incidences from $0.3 \%$ [11] to $1.33 \%$ [10]. This observed variability of occurrence may be in part due to a lack of a clinical registry for VLU [12] and the different methodologies used to collect prevalence and incidence data. As the number of people with VLUs across the globe is

(c) The Author(s). 2021 Open Access This article is licensed under a Creative Commons Attribution 4.0 International License, which permits use, sharing, adaptation, distribution and reproduction in any medium or format, as long as you give appropriate credit to the original author(s) and the source, provide a link to the Creative Commons licence, and indicate if changes were made. The images or other third party material in this article are included in the article's Creative Commons licence, unless indicated otherwise in a credit line to the material. If material is not included in the article's Creative Commons licence and your intended use is not permitted by statutory regulation or exceeds the permitted use, you will need to obtain permission directly from the copyright holder. To view a copy of this licence, visit http://creativecommons.org/licenses/by/4.0/ The Creative Commons Public Domain Dedication waiver (http://creativecommons.org/publicdomain/zero/1.0/) applies to the data made available in this article, unless otherwise stated in a credit line to the data. 
expected to rise in the future due to an aging and an increasingly overweight population $[2,13,14]$, a systematic collation and review of existing prevalence and incidence studies on VLU will inform decision making, priority setting and health care planning as well as future research.

This protocol for a systematic review will employ strict methodological inclusion and exclusion criteria of published and available literature to identify prevalence and incidence of VLU internationally and will characterize the population as reported in these studies.

The following research questions will be addressed:

What is the prevalence of VLUs for different settings according to internationally published studies?

What is the incidence of VLUs for different settings according to internationally published studies?

What are determinants of VLUs in different settings as reported in these studies?

\section{Objectives}

The objective of this systematic review protocol is to present a transparent process. In particular:

- To systematically search the databases to identify studies in which the prevalence and/or incidence of VLU in any care setting in any country are reported

- To describe information sources of the identified studies reporting prevalence and/or incidence of VLU patients

- To extract and appraise the data from the included studies about prevalence and incidence as well as the population characteristics

- To describe the coding procedures as well as the study quality measures and statistical procedures for the quantitative analysis of data from eligible studies

This systematic review is registered at the International Prospective Register of Systematic reviews (PROSPERO) (CRD42020205855). We will disclose any deviations from this protocol. If so, we will update the PROSPERO record accordingly.

\section{Methods}

We developed this protocol according to the Review and Meta-Analysis-Protocols (PRISMA-P) statement $[15,16]$.

\section{Condition}

The condition is the VLU as described by authors using the following:

Population: Adults 18 years of age and older with a VLU. The diagnosis of VLU will be as reported within the studies.
Those with any other chronic wound, e.g., arterial ulcer, diabetic foot ulcer, pressure ulcer, burns, or surgical wounds will be excluded.

\section{Outcome}

The primary outcomes will be period prevalence or point prevalence or cumulative incidence or incidence rate of VLU.

\section{Inclusion and exclusion criteria}

We will include intervention studies and observational studies such as cohort studies, case control studies, cross sectional studies. We will exclude editorials, letters, case studies, case series, and animal studies. Studies will be included regardless of language, sample size, or year of publication.

\section{Information sources}

We will search the following electronic databases: Medline (PubMed), Cumulative Index to Nursing and Allied Health Literature (CINAHL) (EBSCO platform), Embase, Scopus, Web of Science, LiSSa (Littérature Scientifique en Santé), Google Scholar, and Cochrane Database of Systematic Reviews.

\section{Search strategy}

The search strategy will be designed and conducted in collaboration with an experienced reference librarian of the HES-University of Applied Sciences and Arts Western Switzerland, Geneva (MP), in consultation with the authors. To guide the electronic literature search strategies, we will use the Peer Review of Electronic Search Strategies (PRESS) 2015 Guideline Statement [17]. To construct a comprehensive set of possible search terms, we will apply controlled vocabulary (e.g., Medical Subject Headings terms) with keywords both in full and in various truncations (see Table 1). Additionally, we will use Boolean operators and proximity operators, including wildcards, AND, OR, parentheses, and quotations for each database. The initial search strategy was designed and piloted on September 2, 2020, and tested for possible study volume on September 7, 2020. We will run the searches firstly with research design filters and then with extensive qualitative filters applied. Table 2 summarizes the search strategy applied for Medline and CINAHL electronic databases.

\section{Study records}

\section{Data management}

We will import all references into one single EndNote library version X8. Titles will be de-duplicated once entered into EndNote library. We then will export the references from the EndNote Library into the software 
Table 1 Keywords

\begin{tabular}{llll}
\hline Concepts & Keywords & MESH (PubMed) & $\begin{array}{l}\text { CINAHL headings (CINAHL } \\
\text { Complete) }\end{array}$ \\
\hline Venous leg ulcer & "venous leg ulcer" & $\begin{array}{l}\text { "varicose ulcer"[MeSH Terms] } \\
\text { AND "leg ulcer"[MeSH Terms] }\end{array}$ & $\begin{array}{l}\text { (MH "Venous Ulcer") AND } \\
\text { (MH "Leg Ulcer") }\end{array}$ \\
Prevalence OR incidence & $\begin{array}{l}\text { PubMed } \\
\text { prevalence OR incidence OR } \\
\text { occurrence OR epidemiolog* }\end{array}$ & \\
\hline
\end{tabular}

Rayyan. This software will support the screening process.

\section{Selection process}

Two reviewers (SP, PB) being experts in VLU and conducting reviews in this field will independently screen titles and abstracts for those matching the eligibility criteria. We will retrieve the full-texts of the relevant eligible studies. Two reviewers will independently assess the full texts for study characteristics. The excluded studies will be listed in a table including the reason for exclusion. We will resolve any discrepancies between the reviewers involving a third reviewer. Finally, we will prepare a PRISMA-flowchart to document the final selection process.

\section{Methodological quality appraisal}

Two independent reviewers will conduct a risk of bias assessment; any disagreements will be resolved through discussion or consultation with a third reviewer if needed. To assess the methodological quality of the included studies, the quality appraisal tool for systematic reviews of prevalence data will be used [18]. The quality of evidence will be assessed using the Grading of Recommendations Assessment, Development and Evaluation (GRADE) methods [19].

\section{Data extraction}

Included study data will be extracted and managed independently by two reviewers using an electronic data collection form developed by SP, PB, and MP. The information will include study details (e.g., study ID, author, year, journal), study method (e.g., aims of study, setting, study design, outcomes method of data analysis), and results (e.g., prevalence $\mathrm{n} / \mathrm{N}$ (\%), proportion and 95\% confidence intervals $(\mathrm{CI})$, incidence $\mathrm{n} / \mathrm{N}(\%)$, proportion and $95 \% \mathrm{CI}$ and duration of recruitment or the study). Studies in which wounds of various etiologies are reported will only be included if data specific to VLU can be extracted. If data is unclear or missing, we will contact the authors. We will resolve any disagreements between the reviewers through discussion and if needed by involving a third reviewer.

\section{Data synthesis}

We will summarize the study characteristics and findings descriptively and will present these in tabular format. Prevalence, incidence, and the characteristics of the study population will be summarized and synthesized narratively as well as in tables. If possible, odds ratios (for categorical outcome data) or weighted mean differences (for continuous data) and 95\% confidence intervals will be calculated for each included study. To assess the heterogeneity between the studies, we will use the chisquared test [20]. In the case of a heterogeneity, we will carry out a subgroup analysis (e.g., age, sex, and setting) and univariate meta-regression in order to estimate the effect of study-level covariates on the estimates of prevalence and incidence. If we find a high number of sufficiently homogeneous studies (in terms of study design, population, and outcome characteristics), we will perform a meta-analysis. When pooling proportions for meta-analysis, we will use the Logit transformation to calculate the weighted summary proportion under fixed and random effects models [21]. We will then list the proportions, with their $95 \% \mathrm{CI}$, found in the individual studies included in the meta-analysis. We will then present the results graphically in a forest plot. If a metaanalysis is deemed inappropriate, we will present a

Table 2 Search strategy

\begin{tabular}{|c|c|c|c|c|}
\hline Date & Database & Search & $\begin{array}{l}\text { Filters or } \\
\text { limits }\end{array}$ & $\begin{array}{l}\text { Number of } \\
\text { studies }\end{array}$ \\
\hline 07 September 2020 & Medline (PubMed) & $\begin{array}{l}\text { ("venous leg ulcer*" [Title/Abstract] OR ("varicose ulcer"[MeSH Terms] } \\
\text { AND "leg ulcer"[MeSH Terms])) AND ("prevalence"[Title] OR "incidence"[Title] } \\
\text { OR "occurrence"[Title] OR "epidemiolog*"[Title]) }\end{array}$ & None & 54 \\
\hline 07 September 2020 & CINAHL & $\begin{array}{l}\text { (TI "venous leg ulcer*" OR AB "venous leg ulcer*" OR ((MH "Venous Ulcer") } \\
\text { AND (MH "Leg ulcer"))) AND (TI prevalence OR TI incidence OR TI occurrence } \\
\text { OR TI epidemiolog*) }\end{array}$ & None & 21 \\
\hline
\end{tabular}


narrative summary of results as well as in tables/figures, considering the strengths of the studies.

\section{Discussion}

The results of this systematic review will provide key stakeholders with an overview of VLU prevalence, incidence, and VLU determinants. This information will inform healthcare professionals, policy-makers, and consumers in making evidence-based decisions that effectively target and address the VLU burden and will inform future research in this area.

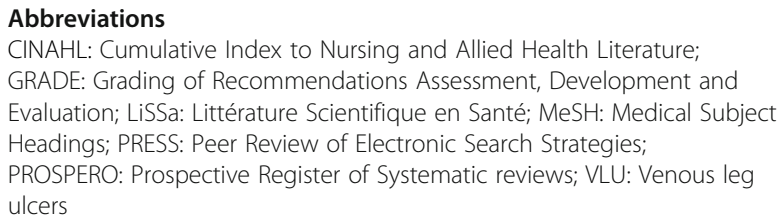

\section{Acknowledgements}

There is no acknowledgement

\section{Authors' contributions}

All authors were responsible and accountable to all part of works related to the protocol. More specifically, SP had the original idea. SP, CD, CS, PB, MBS, and $\mathrm{GG}$ contributed to the conception and design of the protocol. MP performed the first literature searches. SP, CD, CS, PB, MBS, and GG contributed in writing the manuscript. All authors revised the manuscript and gave the approval to the final version to be published.

\section{Funding}

This study is not funded.

\section{Availability of data and materials}

The datasets used and/or analyzed during the current study are available from the corresponding author on reasonable request.

\section{Declarations}

Ethics approval and consent to participate

Not applicable

\section{Consent for publication}

Not applicable

\section{Competing interests}

The authors declare that they have no competing interests.

\section{Author details}

${ }^{1}$ HES-SO University of Applied Sciences and Arts Western Switzerland, School of Health Sciences, Avenue de Champel 47, 1206 Geneva, Switzerland. ${ }^{2}$ Department of Epidemiology and Preventive Medicine, School of Public Health and Preventive Medicine, The Alfred Hospital, Monash University, Melbourne, Australia. ${ }^{3}$ HES-SO University of Applied Sciences and Arts Western Switzerland, Geneva School of Health Sciences, Geneva, Switzerland. ${ }^{4}$ Institute of Epidemiology, Biostatistics and Prevention, Director of Public Health Education Program, University of Zurich, Zurich, Switzerland. ${ }^{5}$ Schoo of Nursing and Midwifery, Aras Moyola, NUI Galway, Galway, Ireland.

Received: 19 October 2020 Accepted: 3 May 2021

Published online: 12 May 2021

\section{References}

1. Cushman M. Epidemiology and risk factors for venous thrombosis. Semin Hematol. 2007:44(2):62-9. https://doi.org/10.1053/j.seminhematol.2007.02.004

2. Nelson EA, Adderley U. Venous leg ulcers. BMJ Clin Evid. 2016;2016:1902.
3. Abbade LP, Lastória S. Venous ulcer: epidemiology, physiopathology, diagnosis and treatment. Int J Dermatol. 2005;44(6):449-56. https://doi.org/1 0.1111/j.1365-4632.2004.02456.x.

4. Frykberg RG, Banks J. Challenges in the treatment of chronic wounds. Adv Wound Care (New Rochelle). 2015;4(9):560-82. https://doi.org/10.1089/ wound.2015.0635.

5. Vasudevan B. Venous leg ulcers: pathophysiology and classification. Indian Dermatol Online J. 2014;5(3):366-70. https://doi.org/10.4103/2229-5178.13 7819.

6. Barnsbee L, Cheng Q, Tulleners R, Lee X, Brain D, Pacella R. Measuring costs and quality of life for venous leg ulcers. Int Wound J. 2019;16(1):112-21. https://doi.org/10.1111/iwj.13000

7. Weller C, Evans S. Venous leg ulcer management in general practice-practice nurses and evidence based guidelines. Aust Fam Physician. 2012; 41(5):331-7.

8. Guest JF, Ayoub N, Mcllwraith T, Uchegbu I, Gerrish A, Weidlich D, et al. Health economic burden that different wound types impose on the UK's National Health Service. Int Wound J. 2017;14(2):322-30. https://doi.org/1 0.1111 /iwj.12603.

9. O'Brien JF, Grace PA, Perry IJ, Burke PE. Prevalence and aetiology of leg ulcers in Ireland. Ir J Med Sci. 2000;169(2):110-2. https://doi.org/10.1007/ BF03166911.

10. Margolis DJ, Bilker W, Santanna J, Baumgarten M. Venous leg ulcer: incidence and prevalence in the elderly. J Am Acad Dermatol. 2002:46(3): 381-6. https://doi.org/10.1067/mjd.2002.121739.

11. Fowkes FGR, Evans CJ, Lee AJ. Prevalence and risk factors of chronic venous insufficiency. Angiology. 2001;52(1_suppl):S5-S15.

12. Fife CE, Walker D, Eckert KA. Qualified clinical data registries: how wound care practitioners can make the most out of the merit-based incentive payment system. Adv Wound Care. 2018;7(11):387-95. https://doi.org/10.1 089/wound.2018.0830

13. Weller CD, Team V, Ivory JD, Crawford K, Gethin G. ABPI reporting and compression recommendations in global clinical practice guidelines on venous leg ulcer management: a scoping review. Int Wound J. 2019;16(2): 406-19. https://doi.org/10.1111/iwj.13048.

14. Berenguer Perez M, Lopez-Casanova P, Sarabia Lavin R, Gonzalez de la Torre $\mathrm{H}$, Verdu-Soriano J. Epidemiology of venous leg ulcers in primary health care: incidence and prevalence in a health centre-a time series study (20102014). Int Wound J. 2019;16(1):256-65. https://doi.org/10.1111/iwj.13026.

15. Moher D, Shamseer L, Clarke M, Ghersi D, Liberati A, Petticrew M, et al. Preferred reporting items for systematic review and meta-analysis protocols (PRISMA-P) 2015 statement. Syst Rev. 2015;4(1):1. https://doi.org/10.1186/204 6-4053-4-1.

16. Munn Z, Moola S, Lisy K, Riitano D, Tufanaru C. Chapter 5: Systematic reviews of prevalence and incidence. In: Aromataris E, Munn Z, editors. JBI Manual for Evidence Synthesis. JBl; 2020. Available from https://synthesisma nual.jbi.global. https://doi.org/10.46658/JBIMES-20-06.

17. McGowan J, Sampson M, Salzwedel DM, Cogo E, Foerster V, Lefebvre C. PRESS Peer Review of Electronic Search Strategies: 2015 Guideline Statement. J Clin Epidemiol. 2016;75:40-6. https://doi.org/10.1016/j.jclinepi.2 016.01.021.

18. Munn Z, Moola S, Riitano D, Lisy K. The development of a critical appraisal tool for use in systematic reviews addressing questions of prevalence. Int J Health Policy Manag. 2014;3(3):123-8. https://doi.org/10.15171/ijhpm.2014.71.

19. Guyatt G, Oxman AD, Akl EA, Kunz R, Vist G, Brozek J, et al. GRADE guidelines: 1. Introduction-GRADE evidence profiles and summary of findings tables. J Clin Epidemiol. 2011;64(4):383-94. https://doi.org/10.1016/j. jclinepi.2010.04.026.

20. Field AP. Meta-analysis of correlation coefficients: a Monte Carlo comparison of fixed- and random-effects methods. Psychol Methods. 2001;6(2):161-80. https://doi.org/10.1037/1082-989X.6.2.161.

21. Sutton AJ, Abrams KR, Jonas M, Sheldon TA, Song F. Methods for metaanalysis in medical research. Chichester, New York: Wiley; 2000.

\section{Publisher's Note}

Springer Nature remains neutral with regard to jurisdictional claims in published maps and institutional affiliations. 